\title{
Navier and Stokes Meet the Wavelet
}

\section{Paul Federbush ${ }^{\star}$}

Department of Mathematics, University of Michigan, Ann Arbor, MI 48109, USA

Received July 8, 1991

Abstract. We work in the space $\mathscr{F}=\mathscr{F}_{\varepsilon}$ of divergence-free measurable vector fields on $R^{3}$ complete in the norm \|\|$^{\prime}$, where

$$
\left(\|v\|^{\prime}\right)^{2}=\operatorname{Sup}_{\substack{x \\ R \leqq 1}}\left(\frac{1}{R}\right)^{1+\varepsilon} \int_{B(x, R)} v^{2}(y) d^{3} y
$$

for some fixed $\varepsilon>0 . B(x, R)$ is the ball of radius $R$ centered at $x$. Given an initial velocity distribution $\vec{v}(0)$ in $\mathscr{F}$, we find $\vec{v}(x, t)$ for $0 \leqq t \leqq T=T\left(\|v(0)\|^{\prime}\right), T>0$, such that $\vec{v}(x, t)$ is the unique strong solution of the Navier-Stokes equations, in a suitable sense. $\left\{\vec{u}_{\alpha}\right\}$

We expand $\vec{v}^{\prime}(x, t)=\vec{v}(x, t)-\vec{v}(x, 0)$ in terms of divergence-free vector wavelets

$$
\vec{v}^{\prime}(x, t)=\sum_{\alpha} c_{\alpha}(t) \vec{u}_{\alpha}(x) .
$$

The Navier-Stokes equations become an infinite set of integral equations for the $c_{\alpha}(t)$. In an appropriate space one realizes the $c_{\alpha}(t)$ satisfying the equations as the fixed point of a contraction mapping. The thus unique solution is the strong solution mentioned above.

Loosely Speaking. Given $\vec{v}(0)$ of finite \|\|$^{\prime}$ norm, there is one and only one $\vec{v}(t)$ of bounded \|\|$^{\prime}$ norm on $[0, T]$ with $T=T\left(\|v(0)\|^{\prime}\right)>0$, that satisfies both

a) the Navier-Stokes equations

and

b) $\lim _{R \rightarrow \infty} \frac{1}{R^{3}} \int_{B(0, R)}[\vec{v}(x, t)-\vec{v}(x, 0)]=\overrightarrow{0}$, all $t$.

^ Supported in part by the National Science Foundation under Grant No. PHY-90-02815 


\section{Section 0. Introduction}

We study the Navier--Stokes equations for an incompressible fluid filling all of $R^{3}$

$$
\begin{aligned}
\frac{\partial \vec{v}}{\partial t}-\Delta \vec{v} & =-(\vec{v} \cdot \vec{\nabla}) \vec{v}-\vec{\nabla} p, \\
\vec{\nabla} \cdot \vec{v} & =0 .
\end{aligned}
$$

In fact we will never see the pressure term in (0.1), since the equations we will deal with ((1.19)) will involve the expression (0.1) integrated against a divergence-free test function. We study the Cauchy problem for initial data $\vec{v}_{0}=\vec{v}(0)$ divergencefree and satisfying

$$
\left(\left\|v_{0}\right\|^{\prime}\right)^{2}=\operatorname{Sup}_{\substack{x \\ R \leqq 1}}\left(\frac{1}{R}\right)^{1+\varepsilon} \int_{B(x, R)} \vec{v}_{0}^{2} d^{3} y<\infty .
$$

One should note that the data may have infinite energy, i.e.

$$
\int \vec{v}_{0}^{2} d^{3} x=\infty
$$

and still satisfy (0.3); Eq. (0.3) implies only that the energy density be bounded. (Our norm is somewhat similar to the Morrey norm used by Giga and Miyakawa in [7].)

We will define a class of solutions of the Navier-Stokes equations ( $\mathscr{C}$-solutions in Subsect. 1.5) within which there exists for some $T=T\left(\left\|v_{0}\right\|^{\prime}\right)>0$ a solution $\vec{v}(x, t)$ on the interval $[0, T]$ satisfying $\vec{v}(0)=\vec{v}_{0}$. Moreover the solution is unique (in the class of $\mathscr{C}$-solutions). We follow the tradition of calling these strong solutions since they are unique.

The theory of finite energy solutions of the Navier-Stokes equations is quite rich and reaches in many directions (see for example the book of Constantin and Foias [4]). Perhaps the main unsettled question is whether smooth solutions can "blow up." Bounds on the Hausdorff measure of the points in space-time where solutions blow up have been obtained in the beautiful work of Scheffer [8] and Caffarelli, Kohn, and Nirenberg [3]. Our present work does not (yet) impinge on the question of blow up. Strong solutions of the Navier-Stokes equations have been obtained for initial data in $L^{3}$ or $H_{1 / 2}$ (see the paper of Foias and Temam [6]). Our norm, \|\|$^{\prime}$, and the norms in $L^{3}$ and $H_{1 / 2}$ are not comparable, but the \|\|$^{\prime}$ norm is - in our view - "usually weaker" even locally. It is close in spirit to the type of bound associated to studying blow up of the Navier-Stokes equations (see for example Theorem D of [3]). Much the same can be said for the results of [7]. In any case our existence theorem, Theorem 2, does add to one's knowledge also in the finite energy (or presumably finite volume) situation. (It would require overcoming some technical difficulties to adapt our technique for boundary value problems.)

Divergence-free vector wavelets (defined in Subsect. 1.2 and developed in [1 and 2]) were an evolutionary product from the incorporation of the renormalization group into constructive quantum field theory. (Some of the important contributors were K. Wilson, J. Glimm, A. Jaffe, G. Gallavotti, K. Gawedzki, A. Kupiainen, T. Balaban, Y. Meyer, G. Battle, and the author.) It is most natural to study the Navier-Stokes equations using these wavelets, and to see if any information is gained beyond that of the more traditional approaches. (The theory of turbulence, 
and numerical studies of turbulence and the Navier-Stokes equations, should also benefit from the utilization of wavelets.)

Basically, then, one expands the velocity field $v^{\prime}(x, t)=v(x, t)-v(x, 0)$ in terms of the wavelet basis $\left\{u_{\alpha}\right\}$,

$$
v(x, t)-v(x, 0)=v^{\prime}(x, t)=\sum_{\alpha} c_{\alpha}(t) u_{\alpha}(x)
$$

(We require the right side to converge to the left side, in $L_{\text {loc }}^{2}$ ) The Navier-Stokes equations become a coupled set of integral equations for the $c_{\alpha}(t)$ (Eq. (1.19)) - as usual it is more convenient to work with integral equations that the o.d.e. one directly obtains. Using a suitable norm, and a small enough time interval, the solution of the integral equations is realized as the fixed point of a contraction mapping. This is the source of uniqueness, and again is in tune with calling our solutions strong solutions.

It is interesting to note that the solution of the Navier-Stokes equation inside the space $\mathscr{F}$ is not unique. If $v(x, t)$ is such a solution of the Navier-Stokes equations, and $c(t)$ any differentiable three dimensional vector function, then $\hat{v}(x, t)$ is also a solution of the Navier-Stokes equations inside the space $\mathscr{F}$, where

$$
\hat{v}(x, t)=v(x+c(t), t)-\dot{c}(t) .
$$

One may view this non-uniqueness as arising from a time dependent uniform gravitational force applied to the system; such a force may be absorbed into the $\nabla p$ term in $(0.1)$.

Theorem $\mathrm{C}$ of Subsect. 1.5 provides one route to achieve uniqueness. Our $\mathscr{C}$-solutions bound the long wavelength components of $v(x, t)-v(x, 0)$ (through $(1.14)$ and $(0.5))$ to achieve uniqueness. This is most natural to do in the context of a wavelet expansion for $v^{\prime}$ (Eqs. (0.5)) rather than for $v$. In fact the formal wavelet expansion for $v$ (unlike that for $v^{\prime}$ ) may not converge to $v$ ! See the discussion in Subsect. 1.6. The points touched upon in this paragraph all relate to the infinite volume we are working in. As a physicist would say, the problem we are studying is interesting at the infra-red end as well as the ultraviolet.

Section 1 contains all the basic definitions, and the statement of results. Section 2 presents several properties of wavelets beyond those listed in Sect. 1. Section 3 and Subsect. 4.3 contain the proofs of Theorem 1 and Theorem 3, respectively. Each of these theorems concerns questions of convergence of wavelet expansions and comparison of different norms used. Sections 4,5 and 6 comprise the proof of Theorem 2, our existence and uniqueness theorem (via a contraction mapping, Theorem 4 of Subsect. 4.1).

As a browsing route through the paper, to be traversed prior to studying details, we recommend reading: Sect. 1, Subsect. 4.1, the Exegesis and Hermeneutics portion at the end of Sect. 5, Subsects. 6.5 and 6.7.

Many questions (that seem accessible) for further study are raised in the paper: the treatment of problems with boundaries, study of weak solutions (see Subsect. 1.5), question of norm continuity of solution (see Subsect. 6.3). To these we add consideration of alternate norms - perhaps say

$$
(\|v\|)^{2}=\operatorname{Sup}_{y} \int_{B(y, 1)} \frac{1}{|x-y|} v^{2}(x) d^{3} x .
$$




\section{Section 1. Definitions, Formalism, Statement of Results}

In this section we define the spaces of functions we work with, and give definitions of several different types of solutions to the Navier-Stokes equations. Theorem 1 is a statement about the relationship between norms we work with, and about convergence of the wavelet expansions. Theorem 2 is our basic uniqueness and existence result. The paper is devoted to a proof of these two theorems. We also state a number of conjectures about the relationship between different types of solutions of the Navier-Stokes equations. These conjectures and some of the formalism given in this section are meant as suggestions for future development.

1.1. Test Functions. $\mathscr{T}$ is our space of test functions, vector fields on $R^{3}$ satisfying:

a) They are divergence-free.

b) They are $\mathscr{C}^{N}$ (for some fixed large $N$ later specified).

c) Each function, and all its derivatives, has exponential fall-off at infinity.

The test functions are defined so as to include our wavelets as examples.

1.2. Wavelets. We work with a set of divergence-free vector wavelets as given in $[1,2]$. We here summarize some of the properties we need of this set, further properties will be given in Sect. 2. (Notation may differ slightly from in [1 and 2].)

Let $\mathscr{W}$ be the set of wavelets. There are vector functions $\psi_{t}, t \in I$, on $R^{3}$. Here $I$ is a finite set.

$$
\mathscr{W}=\left\{2^{(3 / 2) r} \psi_{t}\left(2^{r} x-n\right) \equiv u_{r, \gamma, t}(x)\right\}_{\substack{t \in I \\ n \in Z^{3} \\ r \in Z}}
$$

$\gamma \equiv 2^{-r} n$, and thus is a point on a lattice of edge size $2^{-r}$. Thus the functions $\left\{u_{r, y, t}(x)\right\}$ as $r=0, \pm 1, \pm 2, \ldots, \gamma=2^{-r} n$ with $n$ varying over a unit $3-d$ lattice, and $t$ varying over $\bar{I}$ form our wavelet basis. $u_{r, \gamma, t}(x)$ is "centered" about $\gamma$, and "lives on a length scale" $\ell_{r} \equiv 2^{-r}$. We index the wavelets also by $\alpha$ :

$$
\alpha \leftrightarrow(r, \gamma, t)=(r(\alpha), \gamma(\alpha), t(\alpha))
$$

The set of $u_{\alpha}$ comprising $\mathscr{W}$ satisfy the following properties:

a) The set of $u_{\alpha}$ forms an orthonormal basis for the divergence-free vector fields on $R^{3}$, where the inner product is given as

$$
\left\langle v_{1}, v_{2}\right\rangle=\int d^{3} x \vec{v}_{1} \cdot \vec{v}_{2} .
$$

b) Each $u_{\alpha}$ is $\mathscr{C}^{N}$. (The $N$ is as in Subsect. 1.1.)

c) $\int x^{\rho} u_{\alpha} d^{3} x=0$ for all multi-indices $\rho,|\rho| \leqq N$.

d) $u_{\alpha}$ and each of its derivatives has exponential fall-off at infinity.

\subsection{Some Vector Fields and their Wavelet Expansions.}

Definition. $\mathscr{U}$ is the space of uniformly locally square integrable divergence-free vector fields on $R^{3}$. A locally square integrable vector function of divergence zero is in $\mathscr{U}$ if its \|\|$^{\prime \prime}$ norm is finite, where

$$
\left(\|v\|^{\prime \prime}\right)^{2}=\operatorname{Sup}_{x} \int_{B(x, 1)} v^{2} .
$$


Definition. $\mathscr{F}=\mathscr{F}_{\varepsilon}$ (for some fixed $\varepsilon>0$ ) is the set of functions in $\mathscr{U}$ for which the |l $\|^{\prime}$ norm is finite, where

$$
\left(\|v\|^{\prime}\right)^{2}=\operatorname{Sup}_{\substack{x \\ R \leqq 1}}\left(\frac{1}{R}\right)^{1+\varepsilon} \int_{B(x, R)} v^{2} .
$$

Definition. For a function $v$ in $\mathscr{U}$ we define its $\alpha^{\text {th }}$ expansion coefficient, $c_{\alpha}$, to be

$$
c_{\alpha}(v)=c_{\alpha}=\left\langle u_{\alpha}, v\right\rangle \text {. }
$$

It is easy to see this is a well defined number. (The "inner product" is just the integral of (1.3).)

Definitions. Let $c$ be a set of $\left\{c_{\alpha}\right\}$ (not necessarily arising as expansion coefficients of a function). We will define two norms \|\| and \|\|$_{0}$ on such $c$. But we first must define two auxiliary objects (following (4) and (5) of [5]):

$$
\begin{aligned}
I(x, R) & =\left\{\alpha \mid \gamma(\alpha) \in B(x, R), 2^{-r(\alpha)} \leqq R\right\}, \\
|c|_{x, R}^{2} & =\sum_{\alpha \in I(x, R)} c_{\alpha}^{2} .
\end{aligned}
$$

We then define

$$
\|c\|_{0}^{2}=\operatorname{Sup}_{\substack{x \\ R}}\left(\frac{1}{R}\right)^{P(R)}|c|_{x, R}^{2}
$$

with

$$
P(R)=\left\{\begin{array}{cc}
1+\varepsilon & R \leqq 1 \\
3 & R>1
\end{array}\right.
$$

and

$$
\|c\|^{2}=\operatorname{Max}(S, L)
$$

where

$$
\begin{aligned}
& S=\operatorname{Sup}_{\substack{x \\
R \leqq 1}}\left(\frac{1}{R}\right)^{1+\varepsilon}|c|_{x, R}^{2}, \\
& L=\operatorname{Sup}_{\substack{\alpha \\
r(\alpha)<0}}\left(\frac{1}{\ell_{\alpha}}\right)^{3-\varepsilon} c_{\alpha}^{2} .
\end{aligned}
$$

(Recall $\ell_{\alpha}=2^{-r(\alpha)}$.)

We define $\mathscr{C}$ as the set of $c$ of finite \|\| norm, a normed space in this norm. We let $\mathscr{C}_{0}$ be the set of $c$ of finite \|\|$_{0}$ norm, also a normed space.

Formal Definitions. For a given $v$ we define $c(v)=\left\{c_{\alpha}=\left\langle u_{\alpha}, v\right\rangle\right\}$. For a given $c$ we define $v(c)=\sum_{\alpha} c_{\alpha} u_{\alpha}$.

Theorem 1. a) Let $v$ be in $\mathscr{F}$. Then $c(v)$ is in $\mathscr{C}_{0}$, and one has

$$
\|v\|_{0} \leqq c\|v\|^{\prime} .
$$


(Here and elsewhere we understand $\|v\|_{0}=\|c(v)\|_{0}$, and likewise for the \|\| norm.)

b) Let $c$ be in $\mathscr{C}$. Then $v(c)$ is in $\mathscr{F}$, and one has

$$
\|v(c)\|^{\prime} \leqq c\|c\| \text {. }
$$

For $c$ in $\mathscr{C}, v(c)=\sum c_{\alpha} u_{\alpha}$ converges in $L_{\text {loc }}^{2}$. It is this type of convergence that is always understood.

c) Let $c$ be in $\mathscr{C}$. Then

$$
c(v(c))=c
$$

1.4. Definitions of Continuity. We must define continuity of a path in each of our spaces, $\mathscr{U}, \mathscr{F}$, and $\mathscr{C}$. We will use a definition much weaker than norm continuity. It will be trivial that norm continuity implies continuity in the sense we work with.

Definition. A path $v(t)$ in $\mathscr{U}$,

$$
v(t):[0, T] \rightarrow \mathscr{U}
$$

is continuous if it is uniformly bounded, i.e. $\|v(t)\|^{\prime \prime}<M$, and for each test function $\phi,\langle\phi, v(t)\rangle$ is continuous.

Definition. A path $v(t)$ in $\mathscr{F}$,

$$
v(t):[0, T] \rightarrow \mathscr{F}
$$

is continuous if it is uniformly bounded, and for each test function $\phi,\langle\phi, v(t)\rangle$ is continuous.

Definition. A path $c(t)$ in $\mathscr{C}$,

$$
c(t):[0, t] \rightarrow \mathscr{C}
$$

is continuous if it is uniformly bounded, and with $c(t)=\left\{c_{\alpha}(t)\right\}$, each $c_{\alpha}(t)$ is continuous.

\subsection{Solutions of the Navier-Stokes Equations.}

Definition. $v(t), 0 \leqq t \leqq T$, is a $\mathscr{U}$-solution of the Navier-Stokes equations if it represents a continuous map from $[0, T]$ into $\mathscr{U}$,

$$
v(t):[0, T] \rightarrow \mathscr{U},
$$

and for each test function $\vec{\phi}$ in $\mathscr{T}$, one has

$$
\langle\phi, v(t)\rangle=\left\langle e^{\Delta t} \phi, v(0)\right\rangle-\int_{0}^{t} d \bar{t}\left\langle\partial_{i} e^{\Delta(t-\bar{t})} \phi_{j}, v_{i}(\bar{t}) v_{j}(\bar{t})\right\rangle, \quad 0 \leqq t \leqq T .
$$

In (1.18) it is understood $i$ and $j$ are summed over, and the inner product represents the integral of the functions in it (previously inner products have been only for vector fields).

Definition. $v(t), 0 \leqq t \leqq T$, is an $\mathscr{F}$-solution of the Navier-Stokes equations if it represents a continuous map from $[0, T]$ into $\mathscr{F}$,

$$
v(t):[0, T] \rightarrow \mathscr{F},
$$

and for each $\phi$ in $\mathscr{T}, v$ satisfies Eq. (1.18). 
Definition. $c(t), 0 \leqq t \leqq T$, is a $\mathscr{C}$-solution of the Navier-Stokes equations if it represents a continuous map from $[0, T]$ into $\mathscr{C}$,

$$
c(t):[0, T] \rightarrow \mathscr{C}
$$

and for each $\alpha$ one has:

$$
c_{\alpha}(t)=\left\langle\left(e^{\Delta t}-1\right) u_{\alpha}, v(0)\right\rangle-\int_{0}^{t} d \bar{t}\left\langle\partial_{i} e^{\Delta(t-\bar{t})} u_{\alpha j}, v_{i}(\bar{t}) v_{j}(\bar{t})\right\rangle
$$

where

$$
v(t)=v(0)+\sum c_{\alpha}(t) u_{\alpha} .
$$

Conjecture $A$. (This is certainly true.)

$\mathscr{C}$-solutions are $\mathscr{F}$-solutions.

Conjecture $B$. Given a $v_{0} \in \mathscr{U}$, there is a $T=T\left(\left\|v_{0}\right\|^{\prime \prime}\right)>0$, such that one has a $\mathscr{U}$-solution, $v(t), 0 \leqq t \leqq T$, with $v(0)=v_{0}$.

Theorem 2. Given $v_{0} \in \mathscr{F}$, there is a $T=T\left(\left\|v_{0}\right\|^{\prime}\right)>0$ such that one has a $\mathscr{C}$ solution, $v(t), 0 \leqq t \leqq T$, with $v(0)=v_{0}$. Moreover such a solution is unique.

Note. $\mathscr{F}$-solutions are certainly not unique as pointed out in the introduction. Consider in particular

$$
\vec{v}(x, t)=f(t) \vec{k}
$$

( $\vec{k}$ is the unit vector in the $z$-direction.) For any $f(t)$ these are $\mathscr{F}$-solutions.

Theorem C. (Proof outlined in Subsect. 6.6). If an $\mathscr{F}$-solution satisfies

$$
\lim _{R \rightarrow \infty} \frac{1}{R^{3}} \int_{B(0, R)}[\vec{v}(x, t)-\vec{v}(x, 0)]=\overrightarrow{0}, \quad \text { all } t,
$$

then it equals the corresponding (unique) $\mathscr{C}$-solution.

1.6. Long Wavelength Residues, A Subtlety of the Infinite Volume. Let $w$ be in $\mathscr{U}$. (All we will state will obviously hold with $\mathscr{F}$ and \|\|$^{\prime}$ replacing $\mathscr{U}$ and \|\|$^{\prime \prime}$.) We define its $r$ (level long wavelength) Residue, $w^{r}$, by

$$
w^{r}=w-\sum_{\substack{\alpha \\ r(\alpha) \geqq r}} c_{\alpha} u_{\alpha}
$$

where

$$
c_{\alpha}=\left\langle u_{\alpha}, w\right\rangle \text {. }
$$

This is a somewhat subtle idea. Consider $\vec{w}_{0}=\vec{k}$ : then $w_{0}^{r}=w_{0}$ for all $r, c_{\alpha}=0$, all $\alpha$. (In fact by Theorem 3, part b) below, the only $v$ in $\mathscr{U}$ with all $c_{\alpha}=0$ are constant vectors.) From this example we see the residues do not necessarily go to zero as $r$ approaches $-\infty$. (For $w=v(c), c$ in $\mathscr{C}, \lim _{r \rightarrow-\infty} w^{r}=0$ by Theorem 1.) But in all cases the $w^{r}$ should get smoother and smoother as $r \rightarrow-\infty$. This is made precise by the following theorem. 
Theorem 3. Residue Bounds. For $w$ in $\mathscr{U}$ and $r<0$ we have

a) $\left|w^{r}\right|_{\infty} \leqq c\|w\|^{\prime \prime}$,

b) $\left|D^{\beta} w^{r}\right|_{\infty} \leqq c \frac{1}{\ell_{r}^{|\beta|}}\|w\|^{\prime \prime}$,

c) $\left|\left(\left(e^{\Delta t}-1\right) w\right)^{r}\right|_{\infty} \leqq c t \frac{1}{\ell_{r}^{2}}\|w\|^{\prime \prime}$.

This theorem is treated in Subsect. 4.3.

\section{Section 2. Some Properties of Wavelets}

We continue our discussion of the wavelets introduced in Subsect. 1.2. For their construction see [1 and 2]. The following properties are either explicitly or implicitly from [1 and 2]. (One should note the minor point that $N$ as we use it, beginning in Subsect. 1.2, may differ from the $N$ in [1 and 2].)

Estimate 2.1.

(Recall $\ell_{r}=\ell_{\alpha}=2^{-r}$.)

$$
\left|u_{r, \gamma, t}(x)\right| \leqq c \frac{1}{\left(\ell_{r}\right)^{3 / 2}} e^{-a \frac{|x-y|}{\ell_{r}}}
$$

Estimate 2.2.

$$
\left|D^{\beta} u_{\alpha}\right| \leqq c \frac{1}{\left(\ell_{r}\right)^{3 / 2}} \cdot \frac{1}{\ell_{r}^{|\beta|}} \cdot e^{-a \frac{|x-\gamma|}{\ell_{r}}} \quad|\beta| \leqq N .
$$

Representation 2.3. For $0 \leqq s \leqq N$ there are vector functions $G_{\alpha, \beta}$ such that

$$
u_{\alpha}(x)=\sum_{|\beta|=s}\left(\ell_{\alpha}\right)^{s} D^{\beta} G_{\alpha, \beta}(x) .
$$

Here $\beta$ is a multi-index. Moreover the $G_{\alpha, \beta}$ may be chosen satisfying Estimates 2.1 and 2.2 .

It is important to note that $a$ and $c$ in (2.1) and (2.2) may be picked independent of $\alpha$.

\section{Section 3. Proof of Theorem 1}

In this section we prove Theorem 1. We prove the different parts with varying degrees of thoroughness - but as in the rest of the paper, we are guided largely by the desire to display the different techniques of proof, and arguments, intrinsic to analysis with wavelets.

3.1. Proof of Theorem 1, part a). Let $v$ be in $\mathscr{U}$, then we define two norms on $v$, $|v|_{x, R}^{\prime}$ and $|v|_{x, R}$

$$
\begin{gathered}
\left(|v|_{x, R}^{\prime}\right)^{2}=\int_{B(x, R)} v^{2} \\
|v|_{x, R}=|c(v)|_{x, R} .
\end{gathered}
$$


(See (1.9) for $|c(v)|_{x, R}$.) It is sufficient for a proof of Theorem 1, part a) to prove the following lemma, interesting in its own right.

Lemma. Let $v$ be in $\mathscr{U}$. Then

$$
|v|_{x, R} \leqq c \operatorname{Sup}_{y}|v|_{y, R}^{\prime}
$$

(c an absolute constant).

We proceed to prove this lemma working with a fixed $x$ and $R$.-

Step 1. We now define a decomposition

$$
v=v_{1}+v_{2}
$$

(where $v_{1}$ and $v_{2}$ are not required to be divergence-free)

$$
\begin{aligned}
& v_{1}(y)=\left\{\begin{array}{cl}
v(y) & |x-y|<100 R \\
0 & \text { otherwise }
\end{array},\right. \\
& v_{2}(y)=\left\{\begin{array}{cl}
v(y) & |x-y|>100 R \\
0 & \text { otherwise }
\end{array} .\right.
\end{aligned}
$$

(We note that the choice, 100 , is wasteful; we will be wasteful in estimates.) We have a parallel decomposition of $c_{\alpha}$,

$$
c_{\alpha}=c_{\alpha 1}+c_{\alpha 2}
$$

where

$$
c_{\alpha i}=\left\langle u_{\alpha}, v_{i}\right\rangle, \quad i=1,2
$$

We then have

$$
\begin{aligned}
|v|_{x, R}^{2} & =\sum_{\alpha \in I}\left(c_{\alpha 1}+c_{\alpha 2}\right)^{2} \\
& \leqq 2 \sum_{\alpha \in I}\left(c_{\alpha 1}^{2}+c_{\alpha 2}^{2}\right) \\
& \leqq 2|v|_{x, 100 R}^{\prime 2}+2 \sum_{\alpha \in I} c_{\alpha 2}^{2} .
\end{aligned}
$$

We are abbreviating $I(x, R)$ as $I$, and have used the basic inequality

$$
\int v_{1}^{2} \geqq \sum c_{\alpha 1}^{2}
$$

The first term in $(3.10)$ is $\leqq c\left(\operatorname{Sup}_{y}|v|_{y, R}^{\prime}\right)^{2}$, and we turn to the second term.

Step 2. We consider a rectangular lattice of edge size $\left(\frac{1}{2} R\right)$, i.e. $\left(\frac{1}{2} R\right) Z^{3}$. We label points of the lattice by $i=\vec{i}$. We now decompose the integral for $c_{\alpha 2}$ on the right side of (3.7) into contributions from balls of radius $R$ centered at lattice sides, and apply the Schwartz Inequality to each term in sum getting from (2.1),

$$
\left|c_{\alpha 2}\right| \leqq c \sum_{\substack{i \\ d(x, i)>10 R}}\left(\int_{B(i, R)} v^{2}\right)^{1 / 2} \frac{\ell_{r_{0}}^{3 / 2}}{\ell_{r}^{3 / 2}} e^{-\frac{a|x-i|}{2} \frac{\ell_{r}}{\ell^{2}}},
$$


where $r_{0}$ is such that $R$ and $2^{-r_{0}}$ are as close as possible and $r=r(\alpha)$. It follows that

$$
\left|c_{\alpha 2}\right| \leqq c \operatorname{Sup}_{i}\left(\int_{B(i, R)} v^{2}\right)^{1 / 2} \frac{\ell_{r_{o}}^{3 / 2}}{\ell_{r}^{3 / 2}} e^{-\frac{a \ell_{r_{r}}}{4}} .
$$

Then one has

$$
\sum_{\alpha \in I} c_{\alpha 2}^{2} \leqq c \operatorname{Sup}_{i}\left(\int_{B(i, R)} v^{2}\right) \sum_{r \geqq r_{0}} 2^{3\left(r-r_{0}\right)} 2^{3\left(r-r_{0}\right)} e^{-\frac{a}{2} 2^{r-r_{0}}}
$$

(the second exponential of 2 arises from the sum over $\alpha \in I$, for fixed $r$ ), yielding

$$
\sum_{\alpha \in I} c_{\alpha 2}^{2} \leqq c \operatorname{Sup}_{i} \int_{B(i, R)} v^{2}
$$

This estimate together with (3.10) completes the proof of the lemma.

3.2. Proof of Theroem 1, part b). We are given $c$ in $\mathscr{C}$. We must show $v(c)$ is in $\mathscr{F}$ and

$$
\|v(c)\|^{\prime} \leqq c\|c\|
$$

(for an absolute constant c). This is equivalent to showing, for each $x$ and $R \leqq 1$,

$$
\left(\frac{1}{R}\right)^{\frac{1+\varepsilon}{2}}|v(c)|_{x, R}^{\prime} \leqq c\|c\| \text {. }
$$

We restrict ourself to a fixed $x, R$, and $v$.

Step 1. We now define a decomposition

$$
v=v_{1}+v_{2},
$$

where

$$
v_{1}(y)=\sum_{\alpha \in I(x, 100 R)} c_{\alpha} u_{\alpha}(y)
$$

We get

$$
\begin{aligned}
\left(\frac{1}{R}\right)^{1+\varepsilon} \int_{B(x, R)} v^{2} & \leqq 2\left(\frac{1}{R}\right)^{1+\varepsilon} \int_{B(x, R)} v_{1}^{2}+2\left(\frac{1}{R}\right)^{1+\varepsilon} \int_{B(x, R)} v_{2}^{2} \\
& \leqq 2\left(\frac{1}{R}\right)^{1+\varepsilon} \sum_{\alpha \in I(x, 100 R)} c_{\alpha}^{2}+2\left(\frac{1}{R}\right)^{1+\varepsilon} \int_{B(x, R)} v_{2}^{2} \\
& \leqq c\|v\|^{2}+2\left(\frac{1}{R}\right)^{1+\varepsilon} \int_{B(x, R)} v_{2}^{2} .
\end{aligned}
$$

Step 2. It is enough to show

$$
\left(\frac{1}{R}\right)^{1+\varepsilon} \int_{B(x, R)} v_{2}^{2} \leqq c\|v\|^{2}
$$

to complete the proof. 
We define

(where $\left.I^{c}=I(x, 100 R)^{c}\right)$ and have

$$
v_{2 r}(y)=\sum_{\substack{\alpha \in I^{c} \\ r(\alpha)=r}} c_{\alpha} u_{\alpha}(y)
$$

$$
v_{2}=\sum_{r} v_{2 r}
$$

We now use a "sums to sups" procedure common in constructive quantum field theory. We will define numerical quantities $f_{r}>0$, and note

$$
\left|\sum_{r} v_{2 r}\right|=\left|\sum_{r} f_{r} \frac{1}{f_{r}} v_{2 r}\right| \leqq\left(\sum_{r} f_{r}\right) \operatorname{Sup}_{r} \frac{1}{f_{r}}\left|v_{2 r}\right|
$$

and so

$$
\left(\frac{1}{R}\right)^{1+\varepsilon} \int_{B}\left(\sum_{r} v_{2 r}\right)^{2} \leqq\left(\frac{1}{R}\right)^{1+\varepsilon}\left(\sum_{r} f_{r}\right)^{2} \int_{B}\left(\operatorname{Sup}_{r} \frac{1}{f_{r}}\left|v_{2 r}(y)\right|\right)^{2} .
$$

Define again $r_{0}$ as in (3.12) $\left(2^{-r_{0}} \cong R\right.$ ), and now define the $f_{r}$ :

$$
f_{r}= \begin{cases}\left(\frac{\ell_{r}}{\ell_{r_{0}}}\right)^{t} & r \geqq r_{0} \\ \left(\frac{\ell_{r_{0}}}{\ell_{r}}\right)^{t} & r \leqq r_{0}\end{cases}
$$

with $t>0$ but sufficiently small. Use the estimate (trivial from (1.8)-(1.14) with $D(r)=\frac{1}{2}(1+\varepsilon)$ if $r \geqq 0$, and $D(r)=\frac{1}{2}(3-\varepsilon)$ if $r<0$ :

$$
\left|c_{\alpha}\right| \leqq\|v\| \ell_{r(\alpha)}^{D(r(\alpha))}
$$

to estimate $v_{2 r}$ from $(3.23)$ as

$$
\left|v_{2 r}(y)\right| \leqq\|v\| \ell_{r}^{D(r)} \sum_{\substack{\alpha \in I^{c} \\ r(\alpha)=r}}\left|u_{\alpha}(y)\right| .
$$

One has the easy estimates (for $y$ in $B(x, R)$ )

$$
\left(\sum_{\substack{\alpha \in I^{c} \\ r(\alpha)=r}}\left|u_{\alpha}(y)\right|\right)^{2} \leqq \begin{cases}c \frac{1}{\ell_{r_{0}}^{3}}\left(\frac{\ell_{r}}{\ell_{r_{0}}}\right)^{s o} & r \geqq r_{0} \\ \frac{c}{\ell_{r}^{3}} & r \leqq r_{0}\end{cases}
$$

for any $s_{0}>0$ (c depending on $s_{0}$ ). Putting (3.27), (3.29), and (3.30) into (3.26) and choosing conditions on the parameters

$$
\begin{aligned}
s_{0} & >2 t, \\
t & >0, \\
2 & -\varepsilon-2 t>0, \\
\varepsilon & >2 t,
\end{aligned}
$$


one deduces (3.22) and completes the proof of Theorem 1, part b). One must scribble a few lines on paper, using (3.32) to control the sum $\sum f_{r}$, using (3.31) to control the sup for $r \geqq r_{0}$, and using (3.33) and (3.34) to control the sup for $r \leqq r_{0}$.

3.3. Proof of Theorem 1, Part c). Given $c=\left\{c_{\alpha}\right\} \in \mathscr{C}$, we must show with

$$
v=v(c)=\sum_{\alpha} c_{\alpha} u_{\alpha}
$$

that one has

$$
\left\langle u_{\alpha_{0}}, v\right\rangle=c_{\alpha_{0}} .
$$

Let

$$
\alpha_{0} \leftrightarrow\left(r_{0}, \gamma_{0}, t_{0}\right)
$$

Let

$$
I_{n}=I_{\gamma_{0}, n l_{0}}
$$

(where $\ell_{0}=\ell_{r_{0}}$ ) and

$$
\begin{aligned}
& v_{n}=\sum_{\alpha \in I_{n}} c_{\alpha} u_{\alpha}, \\
& v_{n}^{\prime}=\sum_{\alpha \in I_{n}^{c}} c_{\alpha} u_{\alpha} .
\end{aligned}
$$

$v_{n}$ is in $L_{2}$ and so

$$
\left\langle u_{\alpha_{0}}, v_{n}\right\rangle=c_{\alpha_{0}},
$$

and thus

$$
\left\langle u_{\alpha_{0}}, v\right\rangle=c_{\alpha_{0}}+\left\langle u_{\alpha_{0}}, v_{n}^{\prime}\right\rangle .
$$

We now split $u_{\alpha_{0}}$

$$
u_{\alpha_{0}}=\chi_{n} u_{\alpha_{0}}+\left(1-\chi_{n}\right) u_{\alpha_{0}}=u_{n 1}+u_{n 2} \text {, }
$$

where $\chi_{n}$ is the characteristic function of $B\left(y_{0}, \frac{n}{2} \ell_{0}\right)$. We note that

$$
\left\|v_{n}^{\prime}\right\| \leqq\|v\|,
$$

and so by (2.1) and Theorem 1, part b) one has

$$
\left\langle u_{n 2}, v_{n}^{\prime}\right\rangle \underset{n \rightarrow \infty}{\longrightarrow} 0 \text {. }
$$

So we will be through if we can show

$$
\left\langle u_{n 1}, v_{n}^{\prime}\right\rangle \underset{n \rightarrow \infty}{\longrightarrow} 0
$$

(by (3.42), (3.43), and (3.45))

$$
\left\langle u_{n 1}, v_{n}^{\prime}\right\rangle=\left\langle u_{\alpha_{0}}, \chi_{n} v_{n}^{\prime}\right\rangle .
$$


But $\chi_{n} v_{n}^{\prime}$, supported on $B\left(\gamma_{0}, \frac{n}{2} \ell_{0}\right)$ goes to zero uniformly with $n$ (from which one can deduce (3.46). The estimate of $\chi_{n} v_{n}^{\prime}$ is quite the same as the estimate of $v_{2}$ in Subsect. 3.2. Bounds (3.26)-(3.30) are sufficient to show this.

\section{Section 4. The Contraction Mapping and (Long Wavelength) Residues}

4.1. The Contraction Mapping. We view Eq. (1.19), with (1.20) substituted into it, as of the form

$$
\left\{c_{\alpha}(t)\right\}=F_{T}\left(\left\{c_{\alpha}(t)\right\}, v(0)\right) .
$$

We let $S_{T}$ be the space of continuous functions $c(t)=\left\{c_{\alpha}(t)\right\}, 0 \leqq t \leqq T$, with $c_{\alpha}(0)=0$ and of finite |||| $\mid$ norm:

$$
\left.\||| c_{\alpha}(t)\right\}\left\|\mid=\operatorname{Sup}_{0 \leqq t \leqq T}\right\|\left\{c_{\alpha}(t)\right\} \| .
$$

(The $c(t)$ in $S_{T}$ are continuous paths in the sense of Subsect. 1.4.) $F_{T}$ is then naturally viewed as a mapping of $S_{T}$ into itself. Let $S_{T, M}$ be the subspace of $S_{T}$ satisfying

$$
\text { |I }\left\{c_{\alpha}(t)\right\}\|\| \leqq .
$$

Theorem 4. The Contraction Mapping Theorem. There is an absolute constant $c_{0}$, and a function $F\left(\|v(0)\|^{\prime}, c\right)>0$, such that if $\|v(0)\|^{\prime}<\infty$, and $M=c\|v(0)\|^{\prime}$, with $c>c_{0}$, then, with $T=F\left(\|v(0)\|^{\prime}, c\right), F_{T}$ is a contraction mapping on $S_{T, M}$. Note that by Theorem 1 and Theorem 2, $\|v(t)\|^{\prime}$ is bounded.

We will construct our proof of Theorem 2 by proving Theorem 4 .

4.2. Introduction of the (Long Wavelength) Residues. We now set $v_{0}^{r}$ to be the $r$-residue of $v(0)$ (see Subsect. 1.6). And define

$$
b_{\alpha}=\left\langle u_{\alpha}, v(0)\right\rangle
$$

so that

$$
v(0)=v_{0}^{r}+\sum_{\substack{\alpha \\ r(\alpha) \geqq r}} b_{\alpha} u_{\alpha} .
$$

We now write the expression (1.19) for $c_{\alpha}(t)$ with (1.20) substituted and $v(0)$ replaced by (4.5) with $r=\operatorname{Min}(0, r(\alpha))$ ( $\alpha$ as on the left side of (4.6))

$$
c_{\alpha}(t)=\left\langle\left(e^{\Delta t}-1\right) u_{\alpha}, v(0)\right\rangle-\left[T_{1}+T_{2}+T_{3}+T_{4}\right]
$$

with

$$
\begin{aligned}
& T_{1}=\int_{0}^{t} d \bar{t}\left\langle\partial_{i} e^{\Delta(t-\bar{t})} u_{\alpha j}, v_{0 i}^{r} v_{0 j}^{r}\right\rangle, \\
& T_{2}=\sum_{\alpha^{\prime}}^{t} \int_{0}^{t} d \bar{t}\left\langle\partial_{i} e^{\Delta(t-\bar{t})} u_{\alpha j}, v_{0 i}^{r} u_{\alpha^{\prime} j}\right\rangle\left(b_{\alpha^{\prime}}+c_{\alpha^{\prime}}(\bar{t})\right),
\end{aligned}
$$




$$
\begin{aligned}
& T_{3}=\sum_{\alpha^{\prime}} \int_{0}^{t} d \bar{t}\left\langle\partial_{i} e^{\Delta(t-\bar{t})} u_{\alpha j}, u_{\alpha^{\prime} i}, v_{0 j}^{r}\right\rangle\left(b_{\alpha^{\prime}}+c_{\alpha^{\prime}}(\bar{t})\right) \\
& T_{4}=\sum_{\alpha^{\prime}} \sum_{\alpha^{\prime \prime}} \int_{0}^{t} d \bar{t}\left\langle\partial_{i} e^{A(t-\bar{t})} u_{\alpha j}, u_{\alpha^{\prime} i} u_{\alpha^{\prime \prime} j}\right\rangle\left(b_{\alpha^{\prime}}+c_{\alpha^{\prime}}(\bar{t})\right) \cdot\left(b_{\alpha^{\prime \prime}}+c_{\alpha^{\prime \prime}}(\bar{t})\right)
\end{aligned}
$$

It is understood that in (4.7)-(4.10) the sums include only $b_{\alpha}$ with $r(\alpha) \geqq r$. Interchanging order of summation and integration is not hard to justify.

4.3. Study of the Residues. We turn to consideration of Theorem 3 of Subsect. 1.6. We will work in $\mathscr{F}$ rather than $\mathscr{U}$ in tune with the rest of this paper.

Let $\chi(x)$ be $C^{\infty}$ satisfying

a) $0 \leqq \chi \leqq 1$,

b) $\chi=\left\{\begin{array}{ll}1 & |x|<1 \\ 0 & |x|>2\end{array}\right.$.

We fix $x_{0}$ and $r<0$ and study $w^{r}(x)$ for $x$ near $x_{0}$. We study Theorem 3, part a)

$$
\begin{aligned}
w(x)=w_{1}(x)+w_{2}(x)= & \chi\left(2^{+r-7}\left(x-x_{0}\right)\right) w(x) \\
& +\left(1-\chi\left(2^{+r-7}\left(x-x_{0}\right)\right)\right) w(x), \\
w^{r}= & w_{1}^{r}+w_{2}^{r} .
\end{aligned}
$$

(We define $w_{i}^{r}$ by the same formula as if $w_{i}$ were divergence-free.) Near $x=x_{0}$ we have

$$
w_{2}^{r}=-\sum_{\substack{\alpha \\ r(\alpha) \geqq r}} c_{2 \alpha} u_{\alpha},
$$

where

$$
c_{i x}=\left\langle u_{\alpha}, w_{i}\right\rangle, \quad i=1,2 .
$$

We might be tempted to write

$$
\begin{aligned}
w_{1}^{r} & =w_{1}-\sum_{\substack{\alpha \\
r(\alpha) \geqq r}} c_{1 \alpha} u_{\alpha}, \\
& =\sum_{\substack{\alpha \\
r(\alpha)<r}} c_{1 \alpha} u_{\alpha} \text { (nicht wahr!), }
\end{aligned}
$$

but $w_{1}$ is not divergence-free, so (4.16) does not follow from (4.15) even though $w_{1}$ is in $L^{2}$.

The method we have chosen to handle this problem is to utilize an additional construct. Namely we expand the $\left\{u_{\alpha}\right\}$ to a basis for vector fields (without imposing the divergence-free condition) by expanding the index set $I$ of (1.1). (See Notes below.) We indicate this basis by

$$
\left\{u_{\alpha}\right\} \cup\left\{u_{\alpha}^{g}\right\}
$$


( $g$ for gradient). Then

$$
\begin{aligned}
w_{1}^{r}=w_{1} & -\sum_{\substack{\alpha \\
r(\alpha) \geqq r}} c_{1 \alpha} u_{\alpha}=\sum_{\substack{\alpha \\
r(\alpha)<r}}\left(c_{1 \alpha} u_{\alpha}+c_{1 \alpha}^{g} u_{\alpha}^{g}\right) \\
& +\sum_{\substack{\alpha \\
r(\alpha) \geqq r}} c_{1 \alpha}^{g} u_{\alpha}^{g} .
\end{aligned}
$$

One has the easy estimate, from (2.1), for $r(\alpha)<r$,

$$
\left|c_{1 \alpha}\right| \leqq c\left(\frac{l_{r}^{3}}{l_{r(\alpha)}^{3 / 2}}\right)\|w\|^{\prime}
$$

and the same estimate for $\left|c_{1 \alpha}^{g}\right|$. For the sum in (4.13) one can develop estimates similar to those in (3.29) and (3.30) (this requires a little work). Estimate (4.19) for $c_{1 \alpha}$ and $c_{1 \alpha}^{g}$ handles the first term on the right side of (4.18). For the last term in (4.18) one notes

$$
c_{1 \alpha}^{g}=\left\langle u_{\alpha}^{g}, \chi w\right\rangle=-\left\langle u_{\alpha}^{g},(1-\chi) w\right\rangle
$$

and can analyze this term, thus, as was (4.13). We leave the proof of parts b) and c) of Theorem 3 to the reader.

Note. The additional wavelets are each gradients, and automatically orthogonal to the divergence-free wavelets. Construction of a wavelet basis for gradient vector fields is much simpler than construction of the divergence-free vector fields. They can be constructed with the same properties a)-d) (of (1.3) and (1.4)) and (2.1)-(2.3) with divergence-free replaced by gradient.

Note. An alternate line of proof of Theorem 3 not employing these additional wavelets runs as follows:

$$
\begin{aligned}
w_{1}^{r} & =w_{1}-\sum_{\substack{\alpha \\
r(\alpha) \geqq r}} c_{1 \alpha} u_{\alpha} \\
& =w_{1}+\sum_{\substack{\alpha \\
r(\alpha)<r}} c_{1 \alpha} u_{\alpha}-P w_{1} \\
& =(1-P) w_{1}+\sum_{r(\alpha)<r} c_{1 \alpha} u_{\alpha},
\end{aligned}
$$

where $(1-P)=\frac{K_{u} K_{v}}{K^{2}}$ is projection onto gradient fields, $P$ projection onto divergence-free fields. Working with the explicit form for $(1-P)$ (in $x$-space) one easily gets the same estimates as using the gradient wavelets.

\section{Section 5. Estimation of the Three $\boldsymbol{u}_{\alpha}$ Interaction}

In this section we estimate the "matrix elements"

$$
\left\langle u_{\alpha}, e^{\Delta t}\left(u_{\alpha^{\prime}} \cdot \nabla\right) u_{\alpha^{\prime \prime}}\right\rangle
$$

that appear in (4.10) part of (4.6) the basic equation for $c_{\alpha}(t)$. 
We first note some properties of the "heat kernel" $e^{\Delta t}$, for $t>0$, an integral operator.

$$
\begin{gathered}
e^{\Delta t}(x, y)=\frac{c}{t^{3 / 2}} e^{-\frac{|x-y|^{2}}{4 t}}, \\
\int d^{3} y e^{\Delta t}(x, y)=1 \\
\int d^{3} y\left|D_{x}^{\beta} e^{\Delta t}(x, y)\right| \leqq \frac{c(\beta)}{t^{|\beta| / 2}}
\end{gathered}
$$

We next develop some useful definitions in an obvious abbreviated notation.

$$
M(1,2,3, t)=M(1,2,3)=\left|\left\langle u_{1}, e^{\Delta t} u_{2} \cdot \nabla u_{3}\right\rangle\right| .
$$

We order the r's

$$
\begin{aligned}
\left\{r_{1}, r_{2}, r_{3}\right\} & =\left\{r_{a} \geqq r_{b} \geqq r_{c}\right\}, \\
r_{d} & =\min \left\{r_{1}, r_{3}\right\}, \\
r_{e} & =\max \left\{r_{2}, r_{3}\right\}, \\
r_{f} & =\min \left\{r_{2}, r_{3}\right\}, \\
r_{g} & =\min \left\{r_{e}, r_{1}\right\} .
\end{aligned}
$$

Three $u$ interaction estimate table:

where:

$$
M(1,2,3) \leqq c A B C D E,
$$

$$
\begin{aligned}
& A=\frac{1}{\left(\ell_{1} \ell_{2} \ell_{3}\right)^{3 / 2}}, \\
& B=\frac{1}{\ell_{d}}, \\
& C=\left(\frac{\ell_{a}}{\ell_{b}}\right)^{s}, \quad \text { any } s<N / 2, \\
& D=\frac{\ell_{1}^{s^{\prime}}}{t^{s^{\prime} / 2} \ell_{a}^{3},} \text { any } s^{\prime}<N / 2, \\
& E=e^{-\delta \frac{\left|\gamma_{2}-\gamma_{3}\right|}{\ell_{s}}} \cdot \operatorname{Max}\left\{e^{-\delta \frac{\left|\gamma_{1}-\gamma_{e}\right|}{\ell_{\theta}}}, e^{-\delta \frac{\left|\gamma_{1}-\gamma_{e}\right|^{2}}{t}}\right\}
\end{aligned}
$$

for some smail $\delta$.

To explain how the table arises we detail a single case - the derivation of the table will be seen to be trivial.

Illustrative Case. We set

and

$$
r_{1} \leqq r_{2} \leqq r_{3}
$$

$$
\begin{gathered}
s=s^{\prime}, \\
M(1,2,3)=\left|\left\langle\nabla u_{1}, e^{t \Delta} u_{2} u_{3}\right\rangle\right|,
\end{gathered}
$$


where we have integrated by parts on $\nabla$ and used $\vec{\nabla} \cdot \vec{u}_{2}=0$. We now use Eq. (2.3),

$$
\leqq c\left|\left\langle D^{s+1} G_{1}, e^{t \Delta} u_{2} D^{s} G_{3}\right\rangle\right| \ell_{1}^{s} \ell_{3}^{s},
$$

and integrate by parts again

$$
\leqq c\left|\left\langle D^{s+1+s_{1}} G_{1}, e^{t \Delta}\left(D^{s_{2}} u_{2}\right) G_{3}\right\rangle\right| \ell_{1}^{s} \ell_{3}^{s},
$$

where $\left|s_{1}\right|+\left|s_{2}\right|=|s|$ and we do not indicate the sums over $s$ 's. Another integration by parts:

$$
\begin{aligned}
& \leqq c\left|\left\langle D^{s_{1}+1} G_{1},\left(D^{s} e^{t \Delta}\right)\left(D^{s_{2}} u_{2}\right) G_{3}\right\rangle\right| \ell_{1}^{s} \ell_{3}^{s} \\
& \leqq c\left|\left\langle G_{1}^{\prime},\left(D^{s} e^{t \Delta}\right) G_{2} G_{3}\right\rangle\right| \ell_{1}^{s} \ell_{3}^{s} \frac{1}{\ell_{1}^{s_{1}}+1} \frac{1}{\ell_{2}^{s_{2}}},
\end{aligned}
$$

where $G_{1}^{\prime}$ and $G_{2}$ are implicitly defined, all $G$ 's satisfy (2.1). We let $m$ be the matrix element in (5.23).

$$
M \leqq c|m|\left(\frac{\ell_{3}}{\ell_{2}}\right)^{s} \circ \ell_{1}^{s} \circ \frac{1}{\ell_{1}} .
$$

It now follows from (5.4) and (2.1):

$$
\begin{aligned}
& |m| \leqq c \frac{1}{\left(\ell_{1} \ell_{2} \ell_{3}\right)^{3 / 2}} \cdot \operatorname{Sup}_{z}\left(e^{-\frac{a}{4}\left|z-\gamma_{3}\right| / \ell_{3}} e^{-\frac{a}{4}\left|z-\gamma_{2}\right| / \ell_{2}}\right) \\
& \cdot \operatorname{Sup}\left(e^{-\frac{a}{4}\left|x-\gamma_{1}\right| / \ell_{1}} e^{-\frac{|x-y|^{2}}{8 t}} e^{-\frac{a}{4}\left|y-\gamma_{3}\right| / \ell_{3}}\right) \\
& \cdot \int d^{3} x \int d^{3} y\left|e^{\frac{|x-y|^{2}}{8 t}} D^{s} e^{t \Delta}(x, y)\right| e^{-\frac{a}{2}\left|y-\gamma_{3}\right| / \ell_{3}} \text {. }
\end{aligned}
$$

Equations (5.24) and (5.25) yield the table's estimates using the following simple bounds:

$$
\begin{gathered}
\operatorname{Sup}_{z}\left(e^{-\frac{a}{4}\left|z-\gamma_{3}\right| / \ell_{3}} e^{-\frac{a}{4}\left|z-\gamma_{2}\right| / \ell_{2}}\right) \leqq e^{-\frac{a}{8}\left|\gamma_{2}-\gamma_{3}\right| / \ell_{2}} \\
\operatorname{Sup}_{x, y}\left(e^{-\frac{a}{4}\left|x-\gamma_{1}\right| / \ell_{1}} e^{-|x-y|^{2} / 8 t} e^{-\frac{a}{4}\left|y-\gamma_{3}\right| / \ell_{3}}\right) \\
\leqq \\
\operatorname{Max}\left\{e^{-\frac{a}{8}\left|\gamma_{1}-\gamma_{3}\right| / \ell_{1}}, e^{-\left|\gamma_{1}-\gamma_{3}\right|^{2} / 32 t}\right\} \\
\int d^{3} y e^{-\frac{a}{2}\left|y-\gamma_{3}\right| / \ell_{3}}=c \ell_{3}^{3} \\
\int d^{3} x\left|e^{+|x-y|^{2} / 8 t} D^{s} e^{t \Delta}(x, y)\right| \leqq \frac{c}{t^{s / 2}}
\end{gathered}
$$

Equation (5.29) (similar to (5.4)) can be obtained by a dimensional (or scaling) argument. Likewise with (5.28). Equation (5.26) follows from the fact that either $\left|z-\gamma_{3}\right|$ or $\left|z-\gamma_{2}\right|$ must be greater than $\left|\gamma_{2}-\gamma_{3}\right| / 2$. Equation (5.27) follows from the fact that either $|x-y|$ or $\left(\left|x-\gamma_{1}\right|+\left|y-\gamma_{3}\right|\right)$ must be greater than $\left(\gamma_{1}-\gamma_{3}\right) / 2$ 
The most interesting idea to glean from this section is how (2.3) may be used to study dependence of integrals on levels ( $r$ values).

\section{Exegesis and Hermeneutics.}

To work effectively with wavelets it is more than useful to have rules of thumb: which matrix elements are large, how large are they, how small are the small matrix elements, etc. In this subsection we attempt to explain some of the wisdom of experience, in a hand waving way-not claiming to present every insight one can possess, but the major ones. We will discuss the three $u$ matrix elements of this section, and the two $u$ matrix element analyzed in Sect. 6.1. Everything we say is of course contained in the two tables of estimates; we are trying to "understand" the tables.

We first consider the two $u$ matrix element

$$
M(1,2)=\left|\left\langle u_{1}, e^{\Delta t} u_{2}\right\rangle\right|,
$$

an integral of two $u$ 's

$$
\left|\int d^{3} x u_{1} e^{\Delta t} u_{2}\right|
$$

The question of which matrix elements are large is the analogue of momentum conservation for Fourier transforms (Plancherel's theorem being a special case). For wavelets one does not have so sharp a criterion. We note that $M(1,2)$ is "large" for

$$
\begin{gathered}
r_{1} \cong r_{2}=r \\
\left|\gamma_{1}-\gamma_{2}\right| \leqq c \cdot 2^{-r}
\end{gathered}
$$

and has "size" in this case

$$
M(1,2) \sim\left(\left(\frac{1}{\ell_{r}}\right)^{3 / 2}\right)^{2} \cdot \ell_{r}^{3} \cdot e^{-t / \ell_{r}^{2}},
$$

where the first factors of $\ell_{r}$ arise from estimate (2.1) (the "normalization" of the $u_{\alpha}$ ), the second factor of $\ell_{r}^{3}$ from the integral over $x\left(u_{\alpha}\right.$ "lives" on a cube of side $\left.\ell_{r}\right)$, and the exponential from $e^{t \Delta}$. (Equation (5.32) arises from (6.10), (5.33) from (6.12).) Note that our estimate $e^{-t / \ell_{r}^{2}}$ is a very approximate estimate - but it is more than suggestive.

We find it helpful to represent $M(1,2)$ by a graph (Fig. 1)

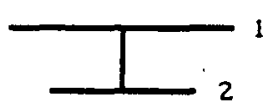

Fig. 1.

where the figure is drawn in the case $\ell_{2} \leqq \ell_{1}$. The $u$ 's are the horizontal lines, the vertical line represents their interaction. We place smaller length scales lower; the three $u$ matrix elements have similar figures. Note that the smaller scale $u_{2}$ must "live" inside the larger scale $u_{1}$ above it.

We turn to the three $u$ matrix element

$$
M(1,2,3)=\mid\left\langle u_{1}, e^{\Delta t} u_{2} \nabla u_{3}\right\rangle .
$$


If we order the $r$ 's as in (5.6),

$$
\left\{r_{1}, r_{2}, r_{3}\right\}=\left\{r_{a} \geqq r_{b} \geqq r_{c}\right\} \text {. }
$$

Then parallel to (5.32) one has for "large" matrix elements

$$
r_{a} \cong r_{b} \geqq r_{c} \text {. }
$$

This follows from (5.14). Thus the figure representing a large matrix will look like Fig. 2.

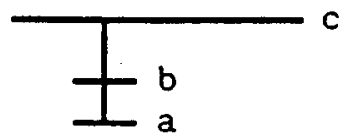

Fig. 2.

where, again, $u_{a}$ must live inside the bigger $u_{c}$ above it. Note that there are

$$
\sim c\left(\frac{\ell_{c}}{\ell_{a}}\right)^{3}
$$

such choices of $u_{a}$, if $r_{a}$ and $u_{c}$ are fixed. Sums over such choices of $u_{a}$ are characteristic of wavelet estimates (such as in deriving (3.14) or (6.29) below).

We first analyze the "size" of the large elements in the case most important to us. (It corresponds to the most delicate situation encountered in analogous situations in quantum field theory.) This proto-type case, Proto-Case 1, is specified by

$$
\begin{gathered}
r=r_{1} \leqq r_{2}=r_{3}=r^{\prime}, \\
\gamma_{2}=\gamma_{3}, \\
\left|\gamma_{1}-\gamma_{2}\right| \leqq 2^{-r},
\end{gathered}
$$

and then

$$
M(1,2,3) \sim\left(\frac{1}{\ell_{r} \ell_{r^{\prime}}^{2}}\right)^{3 / 2} \cdot \ell_{r^{\prime}}^{3} \cdot \frac{1}{\ell_{r}} \cdot e^{-t / \ell_{r}^{2}}
$$

with the factors on the right arising in order as: the normalization of the $u_{\alpha}$, the integral over $x$, the $\nabla$ acting on $u_{1}$ (after integration by parts), and the exponential.

There is one other proto-type, Proto-Case 2 , a situation also where $M(1,2,3)$ is "large,"

$$
\begin{gathered}
r_{1}=r_{3}=r \geqq r_{2}=r^{\prime}, \\
\gamma_{1}=\gamma_{3}, \\
\left|\gamma_{1}-\gamma_{2}\right| \leqq 2^{-r^{\prime}},
\end{gathered}
$$

and then

$$
M(1,2,3) \sim\left(\frac{1}{\ell_{r}^{2} \ell_{r^{\prime}}}\right)^{3 / 2} \cdot \ell_{r}^{3} \cdot \frac{1}{\ell_{r}} \cdot e^{-t / \ell_{r}^{2}} .
$$

$M(1,2,3)$ we must bound will be analyzed as relating to these two proto-cases, or as being irrelevantly small. It is the property of wavelets to allow such localization 
of estimates. One comes to believe: If crude estimates involving only the proto-cases imply convergence, then there is convergence.

\section{Section 6. The Nitty-Gritty}

6.1. Estimation of the Two $u_{\alpha}$ Interaction. In the first few subsections of this section we study the first term on the right side of (1.19). We make several definitions, and collect some relations:

$$
\begin{aligned}
\bar{v}_{0}(t) & =\left(e^{A t}-1\right) v(0), \\
v_{0}(t) & =e^{\Delta t} v(0), \\
\bar{c}_{0 \alpha} & =\left\langle u_{\alpha}, \bar{v}_{0}(t)\right\rangle, \\
c_{0 \alpha} & =\left\langle u_{\alpha}, v_{0}(t)\right\rangle .
\end{aligned}
$$

In this subsection we study, in analogy with Sect. 5:

We define

$$
M(1,2, t)=M(1,2)=\left|\left\langle u_{1}, e^{\Delta t} u_{2}\right\rangle\right| \text {. }
$$

$$
\begin{aligned}
& r_{a}=\max \left\{r_{1}, r_{2}\right\}, \\
& r_{b}=\min \left\{r_{1}, r_{2}\right\}
\end{aligned}
$$

and obtain

$$
M(1,2) \leqq c A B C D
$$

where

$$
\begin{aligned}
& A=\frac{1}{\left(\ell_{1} \ell_{2}\right)^{3 / 2}}, \\
& B=\left(\frac{\ell_{a}}{\ell_{b}}\right)^{s} \text { any } s<N / 2, \\
& C=\frac{\ell_{2}^{s^{\prime}}}{t^{s^{\prime} / 2}} \ell_{a}^{3}, \quad \text { any } s^{\prime}<N / 2, \\
& D=\operatorname{Max}\left\{e^{-\delta \frac{\left|\gamma_{1}-\gamma_{2}\right|}{\ell_{b}}}, e^{-\delta \frac{\left|\gamma_{1}-\gamma_{2}\right|^{2}}{t}}\right\} .
\end{aligned}
$$

This follows as in Sect. 5.

6.2. Bound on $\bar{v}_{0}(t)$. In this subsection we prove the lemma

\section{Lemma.}

$$
\left\|\bar{v}_{0}(t)\right\| \leqq c_{2}\|v(0)\|^{\prime}
$$

We will later pick $c_{0}$ of Theorem 4 (the Contraction Mapping Theorem) to satisfy

$$
c_{0}>c_{2} \text {. }
$$

Now we turn to a proof of (6.13). Referring to (1.12)-(1.14) we see we must show

$$
S \leqq c_{2}\|v(0)\|^{\prime}, \quad L \leqq c_{2}\|v(0)\|^{\prime} .
$$

We first treat $S$. 
It is sufficient to show

$$
\left(\frac{1}{R}\right)^{1+\varepsilon}\left|v_{0}(t)\right|_{x, R}^{2} \leqq c_{a}\left(\|v(0)\|^{\prime}\right)^{2}
$$

using Theorem 1, Part a). We fix $x$ and $R$ through our discussion. We pick $r_{0}$ as small as possible satisfying

$$
2^{-r_{0}} \leqq R \text {. }
$$

We consider a rectangular lattice of edge size $R / 2$, with lattice sites labelled by $i$. Recall

$$
c_{0 \alpha}(t)=\sum_{\alpha^{\prime}} M\left(\alpha, \alpha^{\prime}\right) c_{\alpha^{\prime}}(0)
$$

We split up the sum in (6.17)

$$
=\sum_{i} \sum_{\alpha^{\prime}} M_{i}^{\prime}\left(\alpha, \alpha^{\prime}\right) c_{\alpha^{\prime}}(0)+\sum_{r<r_{0}} \sum_{\alpha^{\prime}} M_{r}^{\prime \prime}\left(\alpha, \alpha^{\prime}\right) c_{\alpha^{\prime}}(0) .
$$

$M_{i}^{\prime}\left(\alpha, \alpha^{\prime}\right)$ is zero unless $\alpha^{\prime} \in I(i, R)$. $M_{r}^{\prime \prime}\left(\alpha, \alpha^{\prime}\right)$ is zero unless $r\left(\alpha^{\prime}\right)=r$. To study $M_{i}^{\prime}$ we use the fact that if $A$ is an operator with matrix elements $A_{i j}$ in an o.n. basis, then

$$
|A|_{0} \leqq \operatorname{Sup}_{i} \sum_{j}\left(\left|A_{i j}\right|+\left|A_{j i}\right|\right),
$$

where ||$_{0}$ indicates operator norm. To study the second term in (6.18) we use the easy bound

$$
\left|c_{\alpha}(0)\right| \leqq\left\{\begin{array}{ll}
c \ell_{r(\alpha)}^{(1+\varepsilon) / 2}\|v(0)\|^{\prime} & r \geqq 0 \\
c \ell_{r(\alpha)}^{3 / 2}\|v(0)\|^{\prime} & r<0
\end{array} .\right.
$$

We now have

$$
\begin{aligned}
\left(\frac{1}{R}\right)^{\frac{1+\varepsilon}{2}}\left|v_{0}(t)\right|_{x, R} \leqq & c \sum_{i}\left|M_{i}^{\prime}\right|_{0}\|v(0)\|^{\prime} \\
& +c \sum_{r<r_{0}}\left(\sum_{\alpha}\left[\sum_{\alpha^{\prime}} M_{r}^{\prime \prime}\left(\alpha, \alpha^{\prime}\right) \frac{\ell_{r}^{Q(r) / 2}}{R^{(1+\varepsilon) / 2}}\right]^{2}\right)^{1 / 2}\|v(0)\|^{\prime},
\end{aligned}
$$

where

From (6.19):

$$
Q(r)=\left\{\begin{array}{cc}
1+\varepsilon & r \geqq 0 \\
3 & r<0
\end{array} .\right.
$$

$$
\left|M_{i}^{\prime}\right|_{0} \leqq c \operatorname{Sup}_{\alpha \in I(x, R)} \sum_{\alpha^{\prime} \in I(i, R)} M\left(\alpha, \alpha^{\prime}\right)
$$

We use (6.8) to estimate the expressions in (6.21) and (6.23).

We split the bound in (6.8) as follows:

$$
\begin{aligned}
A B C D & =\left[A B C\left(\frac{\sqrt{t}}{\ell_{\alpha}}\right)^{s^{\prime} / 2} D^{1 / 2}\right]\left[\left(\frac{\ell_{\alpha}}{\sqrt{t}}\right)^{s^{\prime} / 2} D^{1 / 2}\right] \\
& =N \cdot L .
\end{aligned}
$$


The two brackets in (6.24) define $N$ and $L$. Estimates are expressed with 1 and 2 of (6.5) replaced by $\alpha^{\prime}$ and $\alpha$ respectively. We let

$$
\begin{aligned}
r(\alpha) & =r_{0}, \quad r\left(\alpha^{\prime}\right)=r, \\
r_{a} & =\max \left(r, r_{0}\right), \\
r_{b} & =\min \left(r, r_{0}\right),
\end{aligned}
$$

We claim the following two key estimates:

$$
\begin{aligned}
& \sum_{\substack{\alpha^{\prime} \\
r\left(\alpha^{\prime}\right)=r}} N\left(\alpha, \alpha^{\prime}\right) \leqq\left\{\begin{array}{ll}
\left(\frac{\ell_{a}}{\ell_{b}}\right) & r \geqq r_{0} \\
\left(\frac{\ell_{a}}{\ell_{b}}\right)^{4} & r \leqq r_{0}
\end{array},\right. \\
& \sum_{i} \operatorname{Sup}_{\substack{\alpha \in I(x, R) \\
\alpha^{\prime} \in I(i, R)}} L\left(\alpha, \alpha^{\prime}\right) \leqq c .
\end{aligned}
$$

In (6.29) the sum is over all $\alpha^{\prime}$ of fixed level $r$. We have picked $s=3$, and if $t \geqq \sqrt{\ell_{\alpha}}$ picked $s^{\prime}=6$. This requires $N>12$ (we are certainly wasteful however).

Estimates (6.29) and (6.30) used in analyzing (6.21) and (6.23) yield the bound on $S$. We leave to the reader verifying that (6.8) yields (6.29) and (6.30).

We turn to a treatment of $L$ (from (1.14)). We need show, for $r(\alpha)<0$,

$$
\left|\left\langle u_{\alpha},\left(e^{\Delta t}-1\right) v(0)\right\rangle\right| \leqq c \ell_{r(\alpha)}^{3 / 2-\varepsilon / 2}\|v(0)\|^{\prime} .
$$

We use the fundamental theorem of calculus, often a good idea,

$$
\begin{aligned}
\left\langle u_{\alpha},\left(e^{\Delta t}-1\right) v(0)\right\rangle & =\int_{0}^{t} d \bar{t} \frac{d}{d \bar{t}}\left\langle e^{\Delta \bar{t}} u_{\alpha}, v(0)\right\rangle \\
& =\int_{0}^{t} d \bar{t}\left\langle\Delta u_{\alpha}, e^{\Delta \bar{t}} v(0)\right\rangle \\
& =\sum_{\alpha^{\prime}}^{t} \int_{0}^{t} d \bar{t}\left\langle\Delta u_{\alpha}, e^{\Delta \bar{t}} u_{\alpha^{\prime}}\right\rangle\left\langle u_{\alpha^{\prime}}, v(0)\right\rangle .
\end{aligned}
$$

We use the trivial bound (6.20) for the second factor. The first factor may be treated as in Subsect. 6.1, with the extra $\Delta$ factor yielding $\frac{1}{\ell_{r(x)}^{2}}$ in the bound, the sum over $\alpha^{\prime}$ is as in the treatment of $S$. We get for the matrix element on the left side of (6.32)

$$
\left|\left\langle u_{\alpha},\left(e^{\Delta t}-1\right) v(0)\right\rangle\right| \leqq c \cdot t \cdot \frac{1}{\ell_{r(\alpha)}^{2}} \ell_{r(\alpha)}^{3 / 2}\|v(0)\|^{\prime} .
$$

This easily yields (6.31).

We implicitly will use that $\bar{v}(t)$ is equal to its formal wavelet expansion. This is the statement of Theorem 3, part c).

6.3. Norm Continuity of $v_{0}(t)$ for $t>0$. We consider $0<t_{1}<t_{2}$ and study

$$
\left(e^{\Delta t_{2}}-e^{\Delta t_{1}}\right) v(0)=\int_{t_{1}}^{t_{2}} d t \frac{d}{d t} e^{\Delta t} v(0),
$$


using the fundamental theorem of calculus again as in (6.32). We evaluate the derivative

$$
\int_{t_{1}}^{t_{2}} d t\left[-\frac{3}{2} \frac{1}{t} e^{\Delta t}+\frac{1}{4} \frac{1}{t} \frac{|x-y|^{2}}{t} e^{\Delta t}\right] v(0),
$$

where we understand $\frac{|x-y|^{2}}{t} e^{A t}$ to be the integral operator with kernel

$$
\frac{|x-y|^{2}}{t} e^{A t}(x, y) .
$$

We view (1.12)-(1.14) as realizing \|\| as the maximum of two norms

$$
\|v\|=\operatorname{Max}\left(\|v\|_{s},\|v\|_{L}\right) \text {, }
$$

and write first for $\|v\|_{s}$. Thus we obtain

$$
\begin{aligned}
\left\|\left(e^{\Delta t_{1}}-e^{\Delta t}\right) v(0)\right\|_{s} & \leqq \frac{t_{2}-t_{1}}{t_{1}} \cdot \operatorname{Sup}_{t_{1}<t<t_{2}}\left[c\left\|e^{\Delta t} v(0)\right\|_{s}+c\left\|\frac{|x-y|^{2}}{t} e^{\Delta t} v(0)\right\|_{s}\right] \\
& \leqq \frac{t_{2}-t_{1}}{t_{1}} c\|v(0)\|^{\prime} .
\end{aligned}
$$

This yields the continuity in $S$ norm, for $t>0$. The lemma of Eq. (6.13) controls the first term in brackets in (6.38); the second term in brackets is dealt with the same way - by trivial modification on Subsect. 6.2. We turn to the $L$ norm. Here the same argument as surrounding (6.32) works. We do not investigate whether our solution $v(t)$ is norm continuous for $t>0$.

6.4. Continuity of $v_{0}(t)$ at $t=0$. We need show $c_{0 \alpha}(t)$ is continuous at $t=0$ for each $\alpha$. That is, we must show

$$
\lim _{t \rightarrow 0^{+}}\left\langle u_{\alpha},\left(e^{\Delta t}-1\right) v(0)\right\rangle=0 .
$$

But given $\varepsilon^{\prime}>0$, we can find a bounded set $U$ such that, with $\chi_{u}$ its characteristic function,

$$
\left|\left\langle\left(e^{\Delta t}-1\right) u_{\alpha},\left(1-\chi_{u}\right) v(0)\right\rangle\right|<\varepsilon^{\prime}
$$

by the exponential fall off of $u_{\alpha}$ and $e^{\Delta t}$. Further considering that now $\chi_{u} v(0)$ is in $L^{2}$ one has

$$
\left\langle\left(e^{\Delta t}-1\right) u_{\alpha}, \chi_{u} v(0)\right\rangle \underset{t \rightarrow 0^{+}}{\longrightarrow} 0
$$

(easily seen in Fourier transform space), and our result.

6.5. Heart of the Proof. We turn to the second term on the right side of (1.19) or (4.6) as expanded out in (4.6)-(4.10). We will not prove that the map $F_{T}$ of (4.1) carries continuous paths into continuous paths, but concentrate on showing $F_{T}$ is a contraction if $T$ is small enough.

An Important Reduction (an Application of the Residue Bounds). We make a critical observation:

The contributions of the $v_{0}^{r}$ in (4.6)-(4.10) may be estimated as though they were contributions of $r$-level expansion coefficients! 
Specifically, the term

$$
\left|\left\langle\partial_{i} e^{\Delta(t-\bar{t})} u_{\alpha j}, v_{0 i}^{r} u_{\alpha^{\prime} j}\right\rangle\right|
$$

from (4.8) may be bounded, using the Residue Bounds of Theorem 3 a) and b) $((1.25)-(1.26))$ and the techniques of Sect. 5 as being

$$
\leqq \sum_{\substack{\alpha^{\prime \prime} \\ r\left(\alpha^{\prime \prime}\right)=r}} M^{B}\left(\alpha, \alpha^{\prime \prime}, \alpha^{\prime}\right)\left|c_{\alpha^{\prime \prime}}^{p}\right|
$$

with

$$
\left|c_{\alpha^{\prime \prime}}^{p^{\prime}}\right| \leqq c \ell_{r}^{3 / 2}\left\|v_{0}\right\|^{\prime} .
$$

Here the $\left\{c_{\alpha^{\prime \prime}}^{p}\right\}$ are the "pseudo-coefficients" and $M^{B}$ the bound in (5.11), i.e.

$$
M(1,2,3) \leqq c A B C D E=M^{B}=M^{B}(1,2,3) .
$$

The other terms in $v_{0}^{r}$ are treated similarly. Basically the Residue Bounds give the same estimates, as (2.1)-(2.2) give for the $u_{\alpha}$, to $v_{0}^{r}$ (localized to regions of size $\sim \ell_{r}^{3}$ by a partition of unity) normalized by the $c_{\alpha}^{p}$ to have the right $\left\|v_{0}\right\|^{\prime}$ dependence. Because of (6.45) we modify $M^{B}$ to $\tilde{M}^{B}$ as follows:

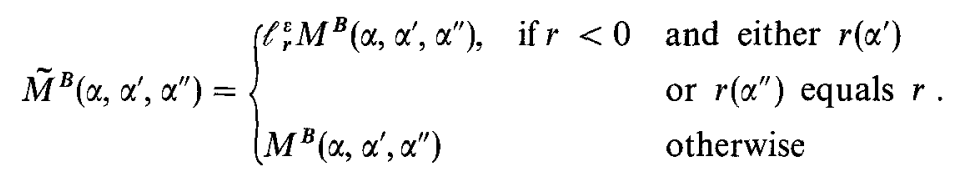

Here $r=r(\alpha)$.

In which Subsubsection the Ultimate Lemma is Introduced. Let $\left\{Y_{\alpha}\right\}$ and $\left\{Z_{\alpha}\right\}$ be vectors in $\mathscr{C}$, and define $\left\{X_{\alpha}(t)\right\}$ by

$$
X_{\alpha}(t)=\sum_{\alpha^{\prime}, \alpha^{\prime \prime}} \tilde{M}^{B}\left(\alpha, \alpha^{\prime}, \alpha^{\prime \prime}, t\right) Y_{\alpha^{\prime}} Z_{\alpha^{\prime \prime}}, \quad 0<t \leqq 1 .
$$

We plan to prove:

\section{The Ultimate Lemma.}

with

$$
\|X(t)\| \leqq c_{u}(t)\|Y\|\|Z\|
$$

$$
\int_{0}^{t} c_{u}(\bar{t}) d \vec{t} \leqq m(t)
$$

and

$$
m(t) \underset{t \rightarrow 0}{\longrightarrow} 0,
$$

where $c_{u}(t)$ and $m(t)$ have no dependence on $Y$ or $Z$.

It is not difficult to see that a complete proof of Theorem 2 now follows from the Ultimate Lemma and the contraction mapping argument of Sect. 4. The right side of (6.49) can be understood as bounding all terms in (4.7)-(4.10), with

$$
\|Y\|,\|Z\| \leqq c\left[\mid\|c(t)\|\|+\| v(0) \|^{\prime}\right] .
$$


6.6. Theorem $C$. In Eq. (1.18) written for the $\mathscr{F}$-solution one lets $\phi=u_{\alpha}$. One thus gets Eq. (1.19) for $c_{\alpha}(t)$, the formal expansion coefficient of $v^{\prime}(t)$. One writes equations analogous to (4.7)-(4.10), but obtained by substituting instead of (1.20), (4.5) the following:

$$
v(t)=v^{r}(t)+\sum_{\substack{\alpha^{\prime} \\ r\left(\alpha^{\prime}\right) \geqq r}}\left(b_{\alpha^{\prime}}+c_{\alpha^{\prime}}(t)\right) u_{\alpha}
$$

$\left(b_{\alpha}\right.$ as in (4.4)) $r=\operatorname{Min}(0, r(\alpha))$. By the same estimates we are pursuing, one gets $c_{\alpha}(t)$ actually in $\mathscr{C}$ (or $S_{T}$ ). Thus if the formal expansion for $v^{\prime},(0.5)$, converges to $v^{\prime}$ in $L_{\text {loc }}^{2}$, we would actually have the unique $\mathscr{C}$-solution. The formal expansion converges (in $L_{\mathrm{loc}}^{2}$ ) to something, $\hat{v}^{\prime}(t)$, and the condition (1.22) ensures

$$
v^{\prime}(t)=\hat{v}^{\prime}(t) \text {. }
$$

One should note that if $v=v(c)$ for $c$ in $\mathscr{C}$, then

$$
\lim _{R \rightarrow \infty} \frac{1}{R^{3}} \int_{B(R)} v d^{3} x=0 .
$$

6.7. A Model Computation. In this subsection we analyze the contribution of Proto-Case $1,(5.39)-(5.42)$, to the right side of (6.49). More particularly we bound $|X(t)|_{x, R}$ for $R \leqq 1$, and thus verify these proto-case terms' contribution to $X(t)$ satisfy the $S$ portion, see (1.13), of the $\|X(t)\|$ bound in the Ultimate Lemma. (If one splits $Y$ and $Z$ into a finite number of pieces, and verifies each piece satisfies the Ultimate Lemma, for both $S$ and $L$, one has verified the Ultimate Lemma.) This is the central ultra-violet aspect of the problem. Before we continue we would like to emphasize two points. First, it was consideration of this model estimate that initially convinced us to try to prove the theorems of this paper, that they should hold. Experience with quantum field theory led to our belief in the pivotal nature of this estimate. Secondly, it is not difficult in retrospect to see from the proof of the full theorem why the model computation is so reliable. We continue with our model computation, recalling the proto-estimate for the proto-terms (from (5.42):

$$
m \sim\left(\frac{1}{\ell_{r}}\right)^{5 / 2} e^{-\frac{1}{\ell_{r}^{2}} t} .
$$

We pick $r_{0}$ as in (6.16). Using the definition of $\tilde{M}^{B}(1,2,3, t)$ from (6.47) and a simple application of the sums to sups procedure (see discussion surrounding (3.27)), what one must show is that (for some small $s$ )

$$
\begin{gathered}
\left(\frac{1}{R}\right)^{1+\varepsilon} \operatorname{Sup}_{r \geqq r_{0}}\left(\frac{\ell_{r_{0}}}{\ell_{r}}\right)^{s} \sum_{\alpha \in I_{r}}\left(\sum_{\alpha^{\prime}, \alpha^{\prime \prime}} \tilde{M}^{B}\left(\alpha, \alpha^{\prime}, \alpha^{\prime \prime}\right) Y_{\alpha^{\prime}} Z_{\alpha^{\prime \prime}}\right)^{2} \\
\leqq c_{u}^{2}(t)\|Y\|^{2}\|Z\|^{2}
\end{gathered}
$$

(and of course it is understood that (6.50) and (6.51) hold). Here $\alpha \in I_{r}$ abbreviates $\alpha \in I(x, R), r(\alpha)=r$. In (6.57) we are only keeping terms satisfying (5.39)-(5.41), and $\tilde{M}^{B}=m$ is given by (6.56) for these. Equation (6.57) is now

$$
\left(\frac{1}{R}\right)^{1+\varepsilon}\left(\frac{\ell_{r_{0}}}{\ell_{r}}\right)^{s} \sum_{\alpha \in I_{r}}\left(\sum_{\alpha^{\prime}, \alpha^{\prime \prime}} m Y_{\alpha^{\prime}} Z_{\alpha^{\prime \prime}}\right)\left(\sum_{\bar{\alpha}^{\prime}, \bar{\alpha}^{\prime \prime}} m Y_{\bar{\alpha}^{\prime}} Z_{\bar{\alpha}^{\prime \prime}}\right) \text {. }
$$


We denote the first expression in parenthesis by $A(\alpha)$ and the second by $B(\alpha)$. Equation (6.58) becomes

$$
\left(\frac{1}{R}\right)^{1+\varepsilon}\left(\frac{\ell_{r_{0}}}{\ell_{r}}\right)^{s} \sum_{\alpha} A(\alpha) B(\alpha) .
$$

In estimating $A(\alpha)$ we view $Y$ and $Z$ in terms of the ||$_{x, R}$ norm and deduce

$$
\begin{aligned}
\sum_{\alpha} A(\alpha) & \leqq c\left(\frac{1}{\ell_{r}}\right)^{5 / 2} e^{-\frac{t}{\ell_{r}^{2}}} \sum_{\alpha^{\prime} \in I} Y_{\alpha^{\prime}} Z_{\alpha^{\prime}} \\
& \leqq c\left(\frac{1}{\ell_{r}}\right)^{5 / 2} e^{-\frac{t}{\ell_{r}^{2}}|Y|_{x, R}|Z|_{x, R}} \\
& \leqq c\left(\frac{1}{\ell_{r}}\right)^{5 / 2} e^{-\frac{t}{\ell_{r}^{2}} R^{1+\varepsilon}}\|Y\|\|Z\| .
\end{aligned}
$$

In estimating $B(\alpha)$ we view $Y$ and $Z$ in terms of the ||$_{\gamma, 2^{-r}}$ norm $(\gamma=\gamma(\alpha))$,

$$
B(\alpha) \leqq c\left(\frac{1}{\ell_{r}}\right)^{5 / 2} e^{-\frac{t}{\ell_{r}^{2}} \ell_{r}^{1+\varepsilon}}\|Y\|\|Z\| .
$$

Putting (6.60) and (6.61) into (6.59) we get

$$
c\left(\frac{\ell_{r_{0}}}{\ell_{r}}\right)^{s} \ell_{r}^{1+\varepsilon} \frac{1}{\ell_{r}^{5}}\|Y\|^{2}\|Z\|^{2} e^{-2 t / \ell_{r}^{2}}
$$

or

$$
\left(\frac{1}{R}\right)^{\frac{1+\varepsilon}{2}}|X(t)|_{x, R} \leqq c\left(\frac{\ell_{r_{0}}}{\ell_{r}}\right)^{s / 2} \ell_{r}^{\varepsilon / 2} \frac{1}{\ell_{r}^{2}}\|Y\|\|Z\| e^{-t / \ell_{r}^{2}}
$$

Pick $s=\varepsilon / 2$

$$
\begin{aligned}
& \leqq c\|Y\|\|Z\| \frac{1}{\ell_{r}^{2}}\left(\ell_{r_{0}} \cdot \ell_{r}\right)^{\varepsilon / 4} e^{-t / \ell_{r}^{2}} \\
& \leqq c\|Y\|\|Z\| l_{r_{0}}^{\varepsilon / 4} \frac{1}{t^{1-\varepsilon / 8}} .
\end{aligned}
$$

In going from (6.64) to (6.65) one has maximized (6.64) over values of $\ell_{r}$ (by setting a derivative equal zero). We have proved for this model calculation, the Ultimate Lemma, (6.49), with

$$
c_{u}(t)=\frac{c}{t^{1-\varepsilon / 8}} .
$$

Note how delicate, or marginal, even this model computation is. One needs $\varepsilon>0$ to make things work. The different treatment of $A(\alpha)$ and $B(\alpha)$ is a slightly clever idea.

6.8. Other Proto-Case Estimates.

A) Proto-case 1 contributions to $\|X(t)\|_{L}$. See (6.37) for introduction of \|\|$_{S}$ and \|\|$_{L}$ norms. Let $\alpha_{0}$ label coefficient with $r\left(\alpha_{0}\right)=r_{0}<0$. We want a bound on the $\alpha_{0}$ component of $X(t), X_{\alpha_{0}}$. We proceed as follows, with sums all understood 
respecting $(5.39)-(5.41)$,

$$
\begin{aligned}
&\left|X_{\alpha_{0}}\right| \leqq \sum_{\alpha^{\prime}, \alpha^{\prime \prime}} \tilde{m}_{B} Y_{\alpha^{\prime}} Z_{\alpha^{\prime \prime}}, \\
& \tilde{m}_{B} \leqq c \frac{1}{\ell_{r_{0}}^{5 / 2}} e^{-t / \ell_{r_{0}}^{2}} \ell_{r_{0}}^{\varepsilon}, \\
& \sum Y_{\alpha^{\prime}} Z_{\alpha^{\prime}} \leqq \sqrt{\sum_{\alpha^{\prime}} Y_{\alpha^{\prime}}^{2}} \sqrt{\sum_{\alpha^{\prime \prime}} Z_{\alpha^{\prime \prime}}^{2}} \leqq \ell_{r_{0}}^{3}\|Y\|_{0}\|Z\|_{0} .
\end{aligned}
$$

See (1.10) and (1.11) for definition of \|\|$_{0}$. Thus we get (using Theorem 1), parts a) and $b)$ )

$$
\left|X_{\alpha_{0}}\right| \leqq c \ell_{r_{0}}^{(1 / 2)+\varepsilon}\|Y\|\|Z\|
$$

So

$$
\frac{1}{\ell_{r_{0}}^{3 / 2-\varepsilon / 2}}\left|X_{\alpha_{0}}\right| \leqq c \frac{1}{\ell_{r_{0}}^{1-3 \varepsilon / 2}}\|Y\|\|Z\| .
$$

Thus

$$
\|X\|_{L} \leqq c\|Y\|\|Z\|
$$

consistent with the same $c_{u}(t)$ as in (6.66).

B) Proto-case 2 contributions to $\|X(t)\|_{L}$. Proceeding similarly as in A),

$$
\begin{gathered}
\tilde{m}_{B} \leqq c \frac{1}{\ell_{r^{\prime}}^{3 / 2}} \frac{1}{\ell_{r_{0}}} \ell_{r_{0}}^{\varepsilon}, \\
\left|X_{\alpha_{0}}\right| \leqq \sum_{\alpha^{\prime}} \tilde{m}_{B} Y_{\alpha^{\prime}} Z_{\alpha_{0}} .
\end{gathered}
$$

(There is an analogous protocase with the roles of $Y$ and $Z$ interchanged.)

$$
\begin{aligned}
& \leqq c \frac{1}{\ell_{r_{0}}^{1-\varepsilon}} \sum_{r^{\prime} \leqq r_{0}} c \frac{1}{\ell_{r^{\prime}}^{3 / 2}}\left(\ell_{r^{\prime}}^{3 / 2-\varepsilon / 2}\|Y\|\right)\left(\ell_{r_{0}}^{3 / 2-\varepsilon / 2}\|Z\|\right) \\
& \leqq c \ell_{r_{0}}^{1 / 2+\varepsilon / 2}\|Y\|\|Z\|,
\end{aligned}
$$

which as in A) yields the same $c_{u}(t)$, of (6.66). Note that one needs the $\varepsilon$ in (1.14) to ensure convergence of the sum over $r^{\prime}$ in (6.75).

C) Proto-case 2 contributions to $\|X(t)\|_{s}$. We have in similar steps, towards computing $|X(t)|_{x, R}, R \sim 2^{-r_{0}}$ :

$$
X_{\alpha} \leqq c\left(\frac{1}{\ell_{r}} \frac{1}{\ell_{r^{\prime}}^{3 / 2}} e^{-t / \ell_{r}^{2}}\right) Z_{\alpha} \ell_{r^{\prime}}^{D\left(r^{\prime}\right)}\|Y\|,
$$

where

$$
D\left(r^{\prime}\right)=\left\{\begin{array}{ll}
\frac{1+\varepsilon}{2} & r^{\prime} \geqq 0 \\
3 / 2-\varepsilon / 2 & r^{\prime}<0
\end{array} .\right.
$$


Thus

$$
\left|X_{\alpha}\right| \leqq \frac{1}{\ell_{r}} e^{-t / \ell_{r}^{2}}\left|Z_{\alpha}\right| \frac{1}{\ell_{r}^{1-\varepsilon / 2}}\|Y\|,
$$

where in going from (6.77) to (6.79) we have performed the sum over $r^{\prime}\left(r^{\prime} \leqq r\right)$. Note again the necessity of both $\varepsilon$ 's in (6.78). From (6.79), using a sums to sups argument, we get

$$
\left(\frac{1}{R}\right)^{\frac{1+\varepsilon}{2}}|X(t)|_{x, R} \leqq \operatorname{Sup}_{r \geqq r_{0}} c\left(\frac{\ell_{r 0}}{\ell_{r}}\right)^{s / 2}\left(\frac{1}{R}\right)^{\frac{1+\varepsilon}{2}}|Z|_{x, R} \frac{1}{\ell_{r}^{2-\varepsilon / 2}} e^{-t / \ell_{r}^{2}}\|Y\| .
$$

This leads to the same estimate as in (6.63).

6.9. Completion of the Proof. We may distinguish three cases for the sum in (6.48)

Case 1.

$$
r(\alpha) \leqq r\left(\alpha^{\prime}\right) \leqq r\left(\alpha^{\prime \prime}\right)
$$

Case 2.

$$
r(\alpha) \geqq r\left(\alpha^{\prime \prime}\right) \geqq r\left(\alpha^{\prime}\right)
$$

Case 3.

$$
r\left(\alpha^{\prime \prime}\right) \geqq r(\alpha) \geqq r\left(\alpha^{\prime}\right) .
$$

There are actually six cases, involving interchange of the conditions on $\alpha^{\prime}$ and $\alpha^{\prime \prime}$ in cases $1,2,3$; but it is easy to see that the estimates for the other three cases are either the same, or more favorable, than for the cases we are considering.

All essential ideas for a proof of the Ultimate Lemma appear in our model computations of the previous two subsections. We consider the contribution of Case 1 of $(6.81)$ to $\|X\|_{S}^{2}$. We consider parallel to (6.58) (with $M=\tilde{M}^{B}$ )

$$
\left(\frac{1}{R}\right)^{1+\varepsilon}\left(\frac{l_{r_{0}}}{l_{r}}\right)^{s} \sum_{\alpha \in I_{r}}\left(\sum_{\alpha^{\prime}, \alpha^{\prime \prime}} M Y_{\alpha^{\prime}} Z_{\alpha^{\prime \prime}}\right)\left(\sum_{\bar{\alpha}^{\prime}, \bar{\alpha}^{\prime \prime}} M Y_{\bar{\alpha}^{\prime}} Z_{\bar{\alpha}^{\prime \prime}}\right),
$$

where the arguments of $M$ are omitted and $\left(\alpha^{\prime}, \alpha^{\prime \prime}\right)$ and $\left(\bar{\alpha}^{\prime}, \bar{\alpha}^{\prime \prime}\right)$ satisfy (6.81) (as hidden restrictions in the sums). As in Subsect. 6.7 we let $A$ and $B$ denote the two expressions in parentheses in (6.84).

We first study $A$. We consider a lattice of edge size $R / 2$, vertices labelled by $i$, and denote $Y^{i}, Z^{i}$ as $Y$ and $Z$ restricted to $I(i, R)$ (only components in this $I$ retained).

$$
A(\alpha) \leqq c \sum_{i^{\prime}, i^{\prime \prime} \alpha^{\prime}, \alpha^{\prime \prime}} M Y_{\alpha^{\prime}}^{i^{\prime}} Z_{\alpha^{\prime \prime}}^{i^{\prime \prime}}
$$

Again agruments in $M$ are suppressed, as are restrictions, (6.81), on sums. For fixed $i^{\prime}, i^{\prime \prime}$ we will estimate contributions to $(6.85)$ in terms of the ||$_{i^{\prime}, R}$ or ||$_{i^{\prime \prime}, R}$ norms of $Y$ and $Z$ respectively. We find a decomposition of $M$

$$
M\left(\alpha, \alpha^{\prime}, \alpha^{\prime \prime}\right) \leqq S\left(\alpha, \alpha^{\prime}, \alpha^{\prime \prime}\right) L\left(\alpha, \alpha^{\prime}, \alpha^{\prime \prime}\right)
$$


such that

$$
\sum_{\substack{i^{\prime}, i^{\prime \prime} \\ i^{\prime} \in I\left(i^{\prime}, R\right) \\ \alpha^{\prime \prime} \in I\left(i^{\prime \prime}, R\right) \\ \alpha \in I_{r}(x, R)}} L\left(\alpha, \alpha^{\prime}, \alpha^{\prime \prime}\right) \leqq c .
$$

For definition of $I_{r}$, see after (6.57).

Then we have

$$
\sum_{\alpha \in I} A(\alpha) \leqq c R^{1+\varepsilon}\|Y\|\|Z\| \cdot \operatorname{Sup}_{i^{\prime}, i^{\prime \prime}} \operatorname{Sup}_{\alpha^{\prime}} \sum_{\alpha^{\prime \prime}} \sum_{\alpha} S\left(\alpha, \alpha^{\prime}, \alpha^{\prime \prime}\right)+\text { Sym. }
$$

We understand in the sums and sups: $\alpha \in I_{r}(x, R), \alpha^{\prime} \in I\left(i^{\prime}, R\right), \alpha^{\prime \prime} \in I\left(i^{\prime \prime}, R\right)$. We have used (6.19) and the + Sym indicates a term with the roles of $\alpha^{\prime}$ and $\alpha^{\prime \prime}$ interchanged (from (6.19)).

We seek a similar decomposition to $(6.86)$ for $M$ in $B(\alpha)$,

$$
M \leqq \bar{S} \bar{L} \text {. }
$$

In this case we work with a lattice of edge size $\ell_{r} / 2$, and measure norms of $Y^{i}, Z^{i}$ in terms of ||$_{i, \ell_{r}}$,

$$
\sum_{\substack{i^{\prime}, i^{\prime \prime} \\ \alpha^{\prime} \in I\left(i^{\prime}, \ell_{r}\right) \\ \alpha^{\prime \prime} \in I\left(i^{\prime \prime}, \ell_{r}\right)}} \bar{L}\left(\alpha, \alpha^{\prime}, \alpha^{\prime \prime}\right) \leqq c .
$$

In this expression $\alpha$ is fixed, in $I_{r}(x, R)$.

$$
B(\alpha) \leqq c \ell_{r}^{1+\varepsilon}\|Y\|\|Z\| \cdot \operatorname{Sup}_{i^{\prime}, i^{\prime \prime}} \operatorname{Sup}_{\alpha^{\prime}} \sum_{\alpha^{\prime \prime}} \bar{S}\left(\alpha, \alpha^{\prime}, \alpha^{\prime \prime}\right)+\text { Sym. }
$$

Again we understand in (6.91), $\alpha \in I(x, R), \alpha^{\prime} \in I\left(i^{\prime}, \ell_{r}\right), \alpha^{\prime \prime} \in I\left(i^{\prime \prime}, \ell_{r}\right)$.

With reasonable choice of $S$ and $\bar{S}$ we get the same estimate (6.65) as in the model situation. One has used the simple inequality: with

one has

$$
f(t)=c \begin{cases}\ell_{r}^{\varepsilon / 4} \frac{1}{\ell_{r}^{2}} & t \leqq \ell_{r}^{2} \\ \ell_{r}^{\varepsilon / 4} \frac{1}{\ell_{r}^{2}}\left(\frac{\ell_{r}^{2}}{t}\right)^{2} & t \geqq \ell_{r}^{2}\end{cases}
$$

$$
f(t) \leqq \frac{c}{t^{1-\varepsilon / 8}}
$$

The expression for $f(t)$ arises in using (5.15). ( $N$ must be sufficiently large for our estimate to hold.) As messy as (6.88) and (6.91) may seem, as one pursues the factors necessary to yield (6.65), it is almost impossible to see how the deviations from the proto-cases could change the form of the estimate-and they don't. The other cases are essentially similar.

\section{References}

1. Battle, G., Federbush, P.: A Note on Divergence-Free Vector Wavelets. Preprint

2. Battle, G., Federbush, P.: Divergence-Free Vector Wavelets. Michigan Mathematical Journal 40, 181 (1993) 
3. Caffarelli, L., Kohn, R., Nirenberg, L.: Partial Regularity of Suitable Weak Solutions of the Navier-Stokes Equations. Comm. Pure Appl. Math. 35, 771 (1982)

4. Constantin, P., Foias, C.: Navier-Stokes Equations. Chicago Lectures in Mathematics, Chicago: University of Chicago Press 1988

5. Federbush, P.: Local Strong Solution of the Navier-Stokes Equations in Terms of Local Estimates. Preprint

6. Foias, C., Temam, R.: Some Analytic and Geometric Properties of the Solutons of the Evolution Navier-Stokes Equations. J. Math. Pures et Appl. 58, 339 (1979)

7. Giga, Y., Miyakawa, T.: Navier-Stokes Flow in $R^{3}$ with Measures as Initial Vorticity and Morrey Spaces. Comm. PDE 14, 577 (1989)

8. Scheffer, V.: Hausdorff Measure and the Navier-Stokes Equations. Comm. Math. Phys. 55, 97 (1977)

Communicated by K. Gawedzki 